\section{- OPEN ACCESS}

\title{
Left ventricular thrombus formation after acute myocardial infarction
}

\author{
Ronak Delewi, ${ }^{1}$ Felix Zijlstra, ${ }^{2}$ Jan J Piek ${ }^{1}$
}

\begin{abstract}
- Additional references are published online only. To view these references please visit the journal online (http://dx.doi.org/ 10.1136/heartjnl-2012-301962).

${ }^{1}$ Department of Cardiology, Academic Medical Center, University of Amsterdam, Amsterdam, the Netherlands ${ }^{2}$ Department of Cardiology, Erasmus Medical Center, Rotterdam, the Netherlands

\section{Correspondence to} Professor Dr Jan J Piek, Department of Cardiology, Academic Medical Center University of Amsterdam, PO Box 22660, 1100 DD Amsterdam, The Netherlands; j.j.piek@amc.uva.nl
\end{abstract}

Cardiovascular disease remains the leading cause of death in western society. Mortality from acute myocardial infarction (AMI) has decreased since the introduction of primary percutaneous coronary intervention (PCI), which has proved to be superior to thrombolytic therapy by demonstrating lower mortality rates and reduced clinical adverse events. Nevertheless, postinfarct complications still lead to morbidity and mortality in a large number of patients.

One of the most feared complications is the occurrence of thromboembolic events (mostly cerebrovascular accidents) due to left ventricular (LV) thrombus formation. The risk of LV thrombus formation is highest during the first 3 months following acute myocardial infarction, but the potential for cerebral emboli persists in the large population of patients with chronic LV dysfunction. Since these thromboembolic events are usually unheralded by warning signs of transient cerebral ischaemia, the only truly satisfactory medical approach is adequate management of these high risk groups. This article discusses the incidence, diagnosis and management of LV thrombus formation after an AMI.

\section{PATHOGENESIS OF LV THROMBUS}

The combination of blood stasis, endothelial injury and hypercoagulability, often referred to as Virchow's triad, is a prerequisite for in vivo thrombus formation. In the presence of LV thrombus formation after AMI, the three components of this triad can also be recognised (figure 1). LV regional wall akinesia and dyskinesia result in blood stasis, often recognised on two dimensional echocardiography by the occurrence of spontaneous LV contrast. Prolonged ischaemia leads to subendocardial tissue injury with inflammatory changes. Finally, patients with an acute coronary syndrome display a hypercoagulable state with, for example, increased concentrations of prothrombin, fibrinopeptide A, and von Willebrand factor, and decreased concentrations of the enzyme responsible for cleaving von Willebrand factor (ADAMTS13). ${ }^{\mathrm{w} 1}$ w2 This triad can result in the formation of $\mathrm{LV}$ thrombus composed of fibrin, red blood cells, and platelets.

LV thrombus can occur within $24 \mathrm{~h}$ after AMI. One study performing serial echocardiographic studies showed that about $90 \%$ of thrombi are formed at a maximum of 2 weeks after the index event. ${ }^{\text {w3 }}$ However, some patients develop a new LV thrombus after discharge, often in association with worsening LV systolic function. Spontaneous or anticoagulant induced resolution is relatively common in LV thrombus formation after AMI. Thrombus seems to disappear more often in patients with apical akinesia than those with apical aneurysm or dyskinesia. ${ }^{1}$

It has been speculated that LV thrombus plays a positive role in the acutely infarcted myocardium, by offering mechanical support to the infarcted myocardium and therefore protecting against LV rupture. ${ }^{\mathrm{w} 4}$ The thrombus becomes firmly attached to its site of origin, enhancing the underlying myocardial scar, limiting potential infarct expansion, and partially restoring the thickness of the myocardial wall. As a consequence, bulging is reduced, resulting in a more effective myocardial contraction. Often, however, expansion of the infarct zone occurs very early after infarction, before the thrombus has time to organise and is able to prevent formation of LV aneurysm and myocardial rupture.

\section{INCIDENCE}

Early data from the prethrombolytic and thrombolytic eras suggest that in the setting of AMI, LV thrombus was present in $7-46 \%$ of patients, most frequently in acute anterior or apical myocardial infarction. $^{2-4}$ w3-w5 Differences in diagnostic techniques, timing of examination and use of antithrombotic treatment cause substantial variation in the reported frequency of thrombus from different series. In addition, it should be noted that the incidence as reported in autopsy studies is consistently higher compared with clinical studies, probably due to better accuracy but also due to patient selection.

Nowadays the reported incidence is lower. This is probably due to (1) more aggressive anticoagulation therapies in the acute phase (eg, the use of heparin, bivalirudin), (2) smaller infarctions, and (3) improved LV remodelling. Although the use of ACE inhibitors is also thought to be associated with improved LV remodelling, the GISSI-3 study found no difference in LV thrombus rates between patients who did and did not receive lisinopril. ${ }^{5}$

There are limited data on the exact frequency of LV thrombus in PCI treated AMI patients. Two studies found LV thrombus formation in $5.4 \%$ and $7.1 \%$ of patients with acute anterior wall myocardial infarctions. ${ }^{\text {w6 }}$ w7 However, these studies were retrospective, non-serial and only assessed LV 


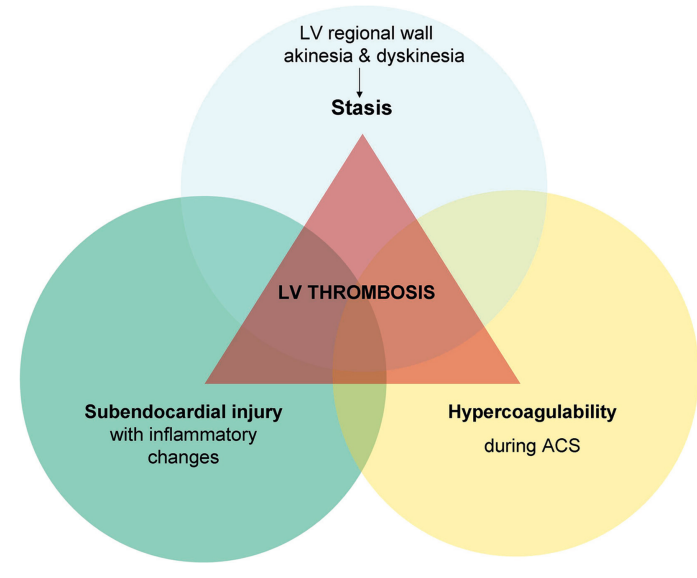

Figure 1 The three components of the Virchow's triad in left ventricular thrombus formation. ACS, acute coronary syndrome; LV, left ventricular.

thrombus formation at a single point in time and during the early phase of recovery after myocardial infarction.

In the latter study a follow-up echocardiography was performed at 1-3 months, showing LV thrombus in an additional $8 \%$ of the patients. ${ }^{\text {w7 }}$ Solheim et al reported a similar incidence of $15 \%$ in the first 3 months in a selected group of AMI patients treated by primary PCI. ${ }^{6}$ So, the timing of LV thrombus assessment is crucial, as assessment too soon after the onset of myocardial infarction will probably lead to failure to detect the thrombus in a significant percentage of patients.

\section{CLINICAL FACTORS CONTRIBUTING TO LV THROMBUS FORMATION}

Risk factors for the development of LV thrombus are consistently irrespective of infarct treatment and include large infarct size, severe apical asynergy (ie, akinesis or dyskinesis), LV aneurysm, and anterior MI. ${ }^{2}{ }^{5-8}$ w6 This is consistent with an increased contribution of at least two of the three components of Virchow's triad, namely a larger area of blood stasis as well as an increased area of injured subendocardium.

In a study of more than 8000 patients with ST elevation myocardial infarction (STEMI), LV thrombus was found in 427 patients (5.1\%). This incidence is relatively low compared to other studies, probably because of the exclusion of high risk patients with severe LV dysfunction. Patients with anterior AMI had a higher incidence of LV thrombus compared to patients with AMI at other regions $(11.5 \%$ vs $2.3 \%, p<0.0001)$. The incidence of LV thrombus was also higher in patients with an ejection fraction $\leq 40 \%$ ( $10.5 \%$ vs $4 \%, p<0.0001)$. In patients with an anterior AMI and an ejection fraction $\leq 40 \%$ this percentage was as high as $17.8 \% .^{5}$

Thrombus formation is not exclusively located apically; approximately $11 \%$ occurs at the septal wall and $3 \%$ at the inferoposterior wall. ${ }^{4}$ The prevalence of thrombus in non-anterior myocardial infarction increases when inferior necrosis extends towards the posterolateral wall. In such cases the prevalence is similar to that observed in anterior wall AMIs of comparable extension. ${ }^{\text {w5 }}$ Thrombi can also be found in small apical infarcts, with good global systolic function. ${ }^{\text {w }}$

The presence of thrombi is significantly related to the region of most severe functional impairment and/or the region with myocardial enhancement (ie, infarction or scarring). $\mathrm{LV}$ thrombus appears earlier in the course of the disease when initial ejection fraction $\leq 40 \%$, in the presence of multivessel coronary artery disease, or a high peak creatine kinase value. ${ }^{\text {w8 }}$

There is conflicting evidence with respect to the influence of $\beta$-blockers. Several studies have reported a higher frequency of thrombus development in patients treated with $\beta$-blockers which could be related to the negative inotropic action of these drugs and thus increased blood stasis. In particular, in a randomised study, Johannessen et al reported an increased occurrence of thrombus in patients with anterior AMI after oral $\beta$-blocker therapy. ${ }^{\mathrm{w} 9}$ Turpie et al reported similar results after treatment with $\beta$-blockers in a large population of patients with AMI. ${ }^{9}$ The GISSI-2 study, however, observed the same rate of LV thrombi in patients with or without atenolol. ${ }^{10}$

It has been demonstrated that mitral regurgitation prevents thrombus formation in patients with dilated cardiomyopathy. ${ }^{\text {w10 }}$ The protective effect of mitral regurgitation may be the consequence of augmented early diastolic flow velocities at the mitral annulus level, as well through the entire length of the left ventricle, protecting the LV cavity from a stagnant, thrombogenic blood flow pattern. In addition, studies suggest abnormal flow profiles are associated with the presence of an LV thrombus. ${ }^{11}$ w11 However, to date no studies have demonstrated the same association in patients with AMI.

There have been few studies on the use of biomarkers in the setting of LV thrombus formation. It could be postulated that factors involved in the coagulation cascade could serve as biomarkers to identify patients at increased risk for LV thrombus development. Data presented at the European Society of Cardiology in 2011 demonstrated higher soluble tissue factor and d-dimer concentrations in patients with LV thrombus formation. ${ }^{\text {w12 }}$ Another study observed mildly elevated anticardiolipin antibody levels in patients with LV thrombus formation after AMI. ${ }^{\mathrm{w} 13}$ Whether these factors are indeed capable of predicting LV thrombus formation needs to be evaluated.

\section{DIAGNOSTIC MODALITIES TO DETECT LV THROMBUS \\ Radionuclide based techniques}

In 39 series using radionuclide ventriculography, a so-called 'square left ventricle' was reported to be associated with LV thrombus. ${ }^{\text {14 }}$ The use of indium-111 labelled platelets is much better documented. It provides excellent specificity (95\%) in 
identifying LV thrombus, and its sensitivity was reported to be $70 \%$ compared with transthoracic echocardiography (TTE). ${ }^{\text {w15 }}$ It is not applied widely though because it is time consuming and expensive, not universally available, and involves radiation exposure. Furthermore, this scintigraphic technique is ineffective in identifying relatively small thrombi, and it has good specificity and sensitivity only if there is active platelet aggregation on the surface of the LV mural thrombus at the time of imaging. In patients with an elevated left hemidiaphragm, indium-111 activity in the spleen may be confused with that from the LV apex. Finally, in patients with a large LV aneurysm but no LV thrombus, a large amount of relatively static blood within the LV aneurysm may increase indium-111 activity. ${ }^{\text {w16 }}$

\section{Echocardiography}

Two dimensional TTE is the technique used most often for assessing the presence, shape and size of an LV mural thrombus. When the thoracic anatomy of the patient allows sufficient visualisation of the heart, two dimensional echocardiography provides excellent specificity (85-90\%) and sensitivity (95\%) in detecting LV thrombus. ${ }^{12}$ w17 w18 LV thrombus on echocardiography is defined as a discrete echodense mass in the left ventricle with defined margins that are distinct from the endocardium and seen throughout systole and diastole. It should be located adjacent to an area of the LV wall which is hypokinetic or akinetic and seen from at least two views (usually apical and short axis). Care must be taken to exclude false tendons and trabeculae and to rule out artefacts (reverberations, side lobe or near field artefacts), which constitute the most common cause for a false diagnosis of a thrombus. ${ }^{13} 14$ Another source of false-positive studies result from tangentially-cut LV wall. Varying gain settings and depth of field, as well as using transducers with different carrier frequencies in multiple positions and orientations, are helpful to minimise such false-positive studies. ${ }^{\text {w17 }}$

In addition, often the LV apex cannot be clearly defined and the presence or absence of a thrombus may be very difficult to establish, leading to an estimated $10-46 \%$ of echocardiograms that are inconclusive. $^{\text {w20 }}$ w21 Intravenous echo contrast during TTE may improve the diagnostic assessment of LV thrombus. ${ }^{12}$ w22 However, in Europe the use of most compounds is contraindicated by the European Medicines Agency in cardiac patients with acute coronary syndromes, recent PCI, acute or chronic severe heart failure or severe cardiac arrhythmias. Also non-protruding and small mural LV thrombi may still go undetected. ${ }^{14}$

Transoesophageal echocardiography (TOE) has little to offer in the detection of LV thrombus. Although it is the technique of choice for detecting atrial masses and thrombi in the left atrial appendage, its value for diagnosing LV thrombus is limited because the apex is most often not well visualised. $^{12}$ w23 Nevertheless, some data suggest that TOE is superior to TTE in providing optimal visualisation of small LV apical thrombi. ${ }^{\text {2 }} 4$

\section{Computed tomography}

CT scanning provides about the same specificity and sensitivity as two dimensional TTE in the identification of LV thrombus. ${ }^{\text {w25 }}$ This technique is not used in daily practice since it requires the intravenous injection of radiographic contrast material and exposes the patient to ionising radiation.

\section{Magnetic resonance imaging}

Cardiac magnetic resonance imaging (CMR) with contrast (delayed enhancement (DE)) has significantly better accuracy than TTE and TOE for the diagnosis of LV thrombus ${ }^{7} 12$ w26 w27 (table 1 and figure 2). A study by Srichai et al compared CMR and late gadolinium enhancement with echocardiography in a cohort of patients undergoing LV reconstruction surgery in whom surgical and/or postmortem verification of thrombus was performed. ${ }^{12}$ This study reported that the sensitivity of TTE was $40 \%$, compared with $88 \%$ for CMR. Another study reported an echo sensitivity and specificity of $33 \%$ and $91 \%$, respectively, in a heterogeneous population of patients with LV systolic dysfunction. ${ }^{7}$ These studies reported lower sensitivity for detection of LV thrombus than previously described, probably due to exclusion of suboptimal echocardiographic examinations in the previously mentioned studies. Also, echocardiographic examinations were often reinterpreted with emphasis on LV thrombus detection and led to different findings when the presence or absence of LV thrombus was based on routine clinical echocardiographic reading as part of the patient's evaluation.

DE-CMR allows for a relatively rapid assessment of thrombus presence, size, and location and is nowadays considered the gold standard. The intravenous administration of gadolinium chelates greatly enhances the ability to detect and characterise LV thrombi. Immediately after contrast administration, the homogeneous, strong enhancement of the LV cavity allows easy detection of abnormal intraventricular structures (dark), which frequently occur adjacent to scarred myocardium (bright hyperenhanced).

Cine-CMR (without a contrast agent such as gadolinium) seems to be less suitable for LV

Table 1 Sensitivities and specificities of different diagnostic modalities for the detection of left ventricular thrombus formation

\begin{tabular}{llc}
\hline & Sensitivity & Specificity \\
\hline TOE & $35 \%$ & $90 \%$ \\
Routine clinical TTE & $35-40 \%$ & $90 \%$ \\
TTE (indication suspect LV thrombus) & $60 \%$ & $90 \%$ \\
CT & Comparable & with TTE \\
Cine CMR & $60 \%$ & $90 \%$ \\
DE-CMR & $88 \%$ & $99 \%$ \\
\hline
\end{tabular}

CMR, cardiac magnetic resonance imaging; $\mathrm{CT}$, computed tomography; $\mathrm{DE}$, delayed enhancement; LV, left ventricular; TOE, transoesophageal echocardiography; TTE, transthoracic echocardiography. 


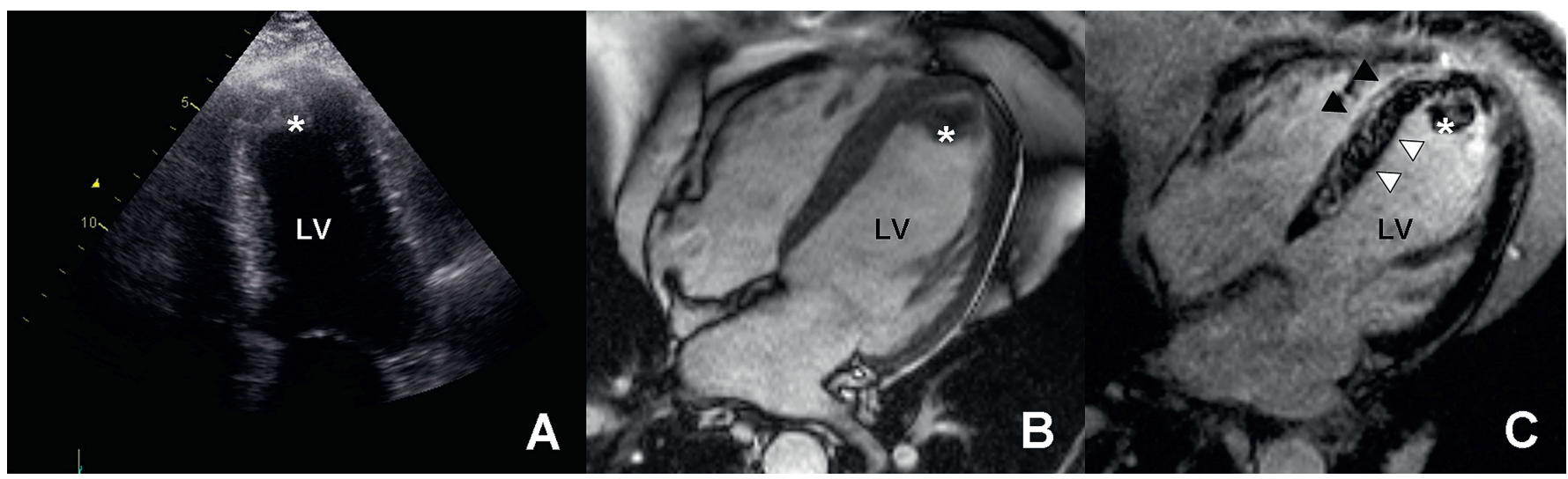

Figure 2 Left ventricular (LV) thrombus formation on delayed gadolinium contrast cardiac MRI and transthoracic echocardiography. Transthoracic echocardiographic appearance of a thrombus (asterisk) in the apex of the left ventricle (A); cine cardiovascular magnetic resonance of the same patient also delineates the apical thrombus $(B)$; late gadolinium enhancement imaging clearly confirms the avascular non-enhancing thrombus (asterisk, dark) close to the transmural infarcted myocardium (bright hyperenhanced, black arrowheads) with areas of microvascular obstruction (black, white arrowheads) (C). Courtesy of Dr A C van Rossum, Dr R Nijveldt, Department of Cardiology, VU University Medical Center, Amsterdam, the Netherlands, and Dr B J Bouma, Department of Cardiology, Academic Medical Center, Amsterdam, the Netherlands.

thrombus detection. Thrombus was missed in $44-50 \%$ of the cases as detected by DE-CMR. ${ }^{7} 8$ The ability of DE-CMR to identify thrombus based on tissue characteristics rather than anatomical appearance alone may explain why it provides improved thrombus imaging compared with cineCMR. It should be mentioned that the criteria to differentiate no-reflow zones from mural thrombi are not definite, and thus differentiation may not always be straightforward. Also, further research and histopathological correlation is needed to evaluate the role of DE-CMR in differentiating subacute from organised clots.

\section{Embolic complications}

In the prethrombolytic era, embolic complications were reported in approximately $10 \%$ of cases, ${ }^{15}$ w28 w29 whereas in the thrombolytic era, embolic complications occurred in $2-3 \%$ of patients. There are poor data regarding embolic complications in LV thrombus patients treated by primary PCI. Also, exact percentages regarding the site of embolisation are not available.

Several studies have suggested that LV thrombi that protrude into the ventricular cavity or that exhibit independent mobility are associated with a higher rate of embolisation than thrombi without these features ${ }^{16} 17$ w30 (figure 3). A thrombus is considered as protruding when it projects predominantly into the LV cavity and as mural when it appears flat and parallel to the endocardial surface. Echocardiographic studies analysing mainly retrospective and non-serial data have indicated a positive relationship between the embolic potential of LV thrombi and their protruding shape and/or intracavitary motion. ${ }^{15}$ w30 However, spontaneous time-course variation in the morphologic aspects, such as shape and mobility pattern, are common. By performing serial echocardiography on 59 untreated patients, Domenicucci et al found that these morphological features demonstrated pronounced spontaneous variability in the first several months after acute infarction, and therefore suggested that the assessment of these features was not helpful. They noted that $41 \%$ of 59 thrombi had significant changes in shape and $29 \%$ had changes in mobility. ${ }^{18}$ Also, it has been reported that up to $40 \%$ of embolism episodes occur in patients whose thrombi are neither protuberant nor mobile.

Other thrombus characteristics, such as thrombus size, ${ }^{16}$ central echolucency ${ }^{17}$ or hyperkinesia of the myocardial segments adjacent to the thrombus, ${ }^{4}$ were found in some studies to be associated with an increased risk of embolism, but were not confirmed by others.

Other conditions that increase the risk of systemic embolisation are: (1) severe congestive heart failure, (2) diffuse LV dilatation and systolic dysfunction, (3) previous embolisation, (4) atrial fibrillation, and (5) advanced patient age. It has been suggested that the risk of embolisation is lower in patients with LV aneurysm, since the absence of LV contraction near the site of the thrombus makes dislodgement unlikely. ${ }^{\text {w31 }}$

\section{PHARMACOLOGICAL MANAGEMENT}

If indeed systemic embolisation is the highest risk of LV thrombus, the central question arises as to how these patients should be treated to prevent embolisation. In the past, if recurrent systemic emboli developed despite anticoagulant therapy, surgical removal of the thrombus was considered necessary. ${ }^{17}$ w31 w32 Nowadays antithrombotic therapy is thought to prevent embolic complications of LV thrombus.

\section{Thrombolysis}

Vaitkus and Barnathan pooled the data from six studies comprising a total of 390 patients and assessed the incidence of LV thrombus formation in those patients treated with thrombolysis versus those without thrombolytic therapy. They were not able to demonstrate a statistical difference in the incidence of LV thrombus formation, only a trend in favour of thrombolysis. ${ }^{19}$ These studies 


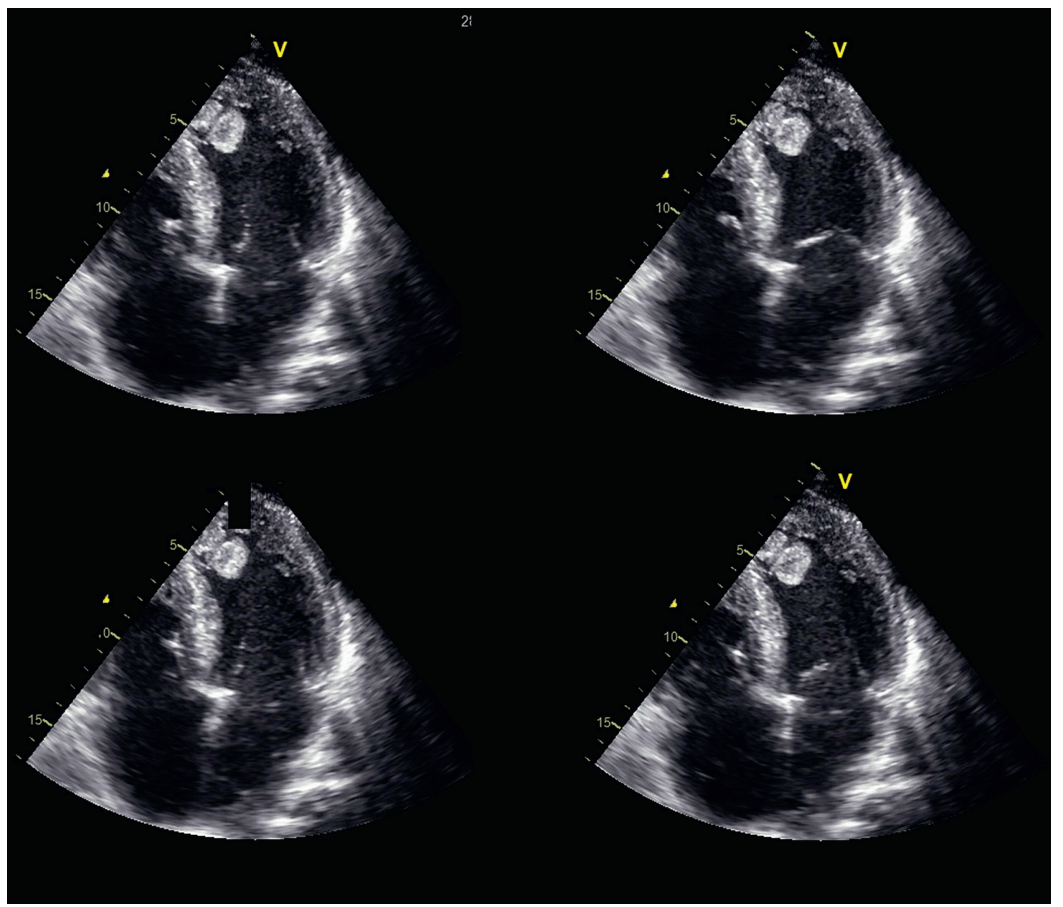

Figure 3 Transthoracic echocardiographic appearance of a mobile, protruding left ventricular thrombus. Courtesy of $\mathrm{J}$ Vleugels and Rianne $\mathrm{H}$ A de Bruin, Department of Cardiology, Department of Cardiology, Academic Medical Center, Amsterdam, the Netherlands. ously every $12 \mathrm{~h}$ ) showed a lower incidence of LV thrombus formation than those administered a low dose (5000 units subcutaneously every $12 \mathrm{~h}$ ) $(11 \%$ vs $32 \%, p<0.001)$ during a 10 day period. ${ }^{9}$ Results from the SCATI study showed a similar reduction in LV thrombus formation for the group that was treated with calcium-heparin compared to the control group in patients undergoing thrombolysis. ${ }^{\text {w34 }}$ In the GISSI-2-connected study, however, high dose heparin did not prevent thrombus formation $(27 \%$ vs $30 \%, p=N S) .{ }^{10}$ In a study with 23 consecutive patients with mobile and protruding thrombi, high dose heparin was given intravenously over a period of 14-22 days (mean 14 4 ). In all 23 patients LV thrombi decreased in size, with disappearance of the high risk features. No embolic events were detected during treatment, and the only complication was an upper gastrointestinal haemorrhage. ${ }^{\text {w35 }}$ Dalteparin, a low molecular weight heparin, reduced the incidence of LV mural thrombus formation but had no influence on the risk of systemic embolisation, and its use was associated with an increased risk of haemorrhage. ${ }^{\text {w36 }}$

\section{Vitamin K antagonist}

Observational studies conducted in the prethrombolytic and thrombolytic eras have provided support for the hypothesis that anticoagulation reduces the risk of embolisation. ${ }^{1} 24$ w3 w37-w39 A 1993 meta-analysis included 11 studies of 856 patients who had an anterior myocardial infarction; the odds ratio (OR) for an embolic event was 5.5 (95\% CI 3.0 to 9.8$).{ }^{19}$ The meta-analysis included seven studies with 270 patients that included data on the relationship between anticoagulation for 6 months and embolisation. Although all seven studies presented data suggesting that systemic anticoagulation reduces embolic complications, this trend reached significance only in three trials. When pooling the data, anticoagulation compared with no anticoagulation was associated with a reduction in the rate of embolisation (OR $0.14,95 \%$ CI 0.04 to 0.52 ).

Based on these data, both current European Society of Cardiology and American College of Cardiology/American Heart Association guidelines recommend vitamin $\mathrm{K}$ antagonist therapy in patients with an LV thrombus after myocardial infarction. ${ }^{\text {4 } 40}$ w41

However, vitamin $\mathrm{K}$ antagonists do not appear to affect the likelihood of resolution of the thrombus $^{\mathrm{w} 3}$ and, unfortunately, no large randomised trials have been performed to evaluate the efficacy of long term anticoagulation to prevent embolisation in patients with LV thrombus. Therefore the effects of long term anticoagulants on the risk of embolisation are the subject of debate. Among the many questions left unanswered is when to withdraw anticoagulant medication when thrombus is identified since the risk of embolisation decreases over time, likely as a result of organisation of thrombus which includes thrombus neovascularisation. However, retrospective studies documented ongoing embolic a benefit, at least in the short term. In a randomised controlled trial, AMI survivors who were treated with high dose heparin (12500 units subcutane- 
Left ventricular thrombus formation after myocardial infarction: key points

- Left ventricular (LV) regional wall akinesia and dyskinesia resulting in blood stasis, prolonged ischaemia leading to subendocardial tissue injury with inflammatory changes and a hypercoagulable state, are consistent with Virchow's triad, resulting in LV thrombus formation.

- Risk factors for the development of LV thrombus include:

- large infarct sizes

- severe apical asynergy

- LV aneurysm

- anterior myocardial infarction

- There is reported controversy regarding the negative influence of $\beta$-blockers and the protective effect of mitral regurgitation.

- Early data from the prethrombolytic and thrombolytic era suggest that in the setting of acute myocardial infarction, LV thrombus was present in $7-46 \%$ of patients.

- Nowadays the reported incidence is lower, probably due to (1) more aggressive anticoagulation therapies in the acute phase (eg, use of heparin, bivalirudin), (2) smaller infarctions, and (3) improved LV remodelling.

- Timing of LV thrombus assessment is crucial, as assessment too soon after the onset of myocardial infarction will miss LV thrombus formation.

- Transthoracic echocardiography is most often used for assessing LV thrombus. However, it is estimated that $10-46 \%$ of echocardiograms are inconclusive.

- Delay enhancement cardiac magnetic resonance imaging (CMR) is nowadays considered the gold standard.

- Cine-CMR, transoesophageal echocardiography, radionuclide angiography, and CT seem less appropriate for LV thrombus detection.

- Conditions that increase the risk of systemic embolisation in patients with LV thrombus are: (1) severe congestive heart failure, (2) diffuse LV dilatation and systolic dysfunction, (3) previous embolisation, (4) advanced age, and (5) presence of LV protruding or mobile thrombi.

- Observational studies conducted in the prethrombolytic and thrombolytic eras have provided support for the hypothesis that warfarin reduces the risk of embolisation. risk in LV thrombus patients. ${ }^{\mathrm{w}}{ }^{42}$ In indium-111 platelet imaging studies most thrombi, regardless of age, have been observed to have externally detectable ongoing platelet accumulation, indicating continued surface activity. ${ }^{20}$ The European guidelines recommend vitamin $\mathrm{K}$ antagonist for at least 3-6 months, while the American guidelines recommend indefinite treatment in patients without increased risk of bleeding.

Although there are limited data regarding the appropriate follow-up and timing of cessation of vitamin $\mathrm{K}$ antagonists in these patients, the following approach seems appropriate for most patients:

- Assess LV thrombus within the first month after AMI, preferably with CMR in high risk patients, and start vitamin $\mathrm{K}$ antagonist when LV thrombus is present and no contraindication exists

- Re-evaluate LV thrombus formation after 6 months since data show that LV thrombus resolution in the initial months is very common, also in patients treated with vitamin $\mathrm{K}$ antagonists $^{\text {w43 }}$

- When LV thrombus is not present and there is no other indication for vitamin $\mathrm{K}$ antagonist, assess bleeding risk and consider stopping therapy.

Newer anticoagulants are presently being developed and some of them are already registered. ${ }^{\mathrm{w} 44-\mathrm{w} 46}$ It can be envisioned that in the longer term these new anticoagulants will replace vitamin $\mathrm{K}$ antagonists. However, at present vitamin $\mathrm{K}$ antagonist therapy is still the standard of care for the treatment of LV thrombus. More importantly, the newer anticoagulants also have the risk of fatal and non-fatal bleedings and their role in LV thrombus patients should be further assessed.

\section{Antiplatelet therapy and triple therapy in the $\mathrm{PCl}$ era}

Another issue is that nowadays STEMI patients are treated by primary PCI and receive long term dual antiplatelet therapy (including aspirin and a P2Y 12 inhibitor). Consequently, patients with LV thrombus or at increased risk of LV thrombus after a myocardial infarction are frequently being treated with vitamin $\mathrm{K}$ antagonist in addition to dual antiplatelet therapy (triple antithrombotic therapy) and therefore are subjected to an increased bleeding risk. It is unclear, however, if long term anticoagulation is still necessary in STEMI patients treated by primary PCI and subsequent dual antiplatelet therapy.

Large prospective studies show a yearly incidence of bleeding of approximately $3.7 \%$ for dual antiplatelet therapy and $12 \%$ for triple antithrombotic therapy. ${ }^{\mathrm{w} 47}$ The most common site of bleeding is the gastrointestinal tract $(30-40 \%)$ and cerebrum (9-10\%), with $25 \%$ of episodes in the latter site proving fatal. Furthermore, non-fatal bleedings are an important predictor of mortality post-PCI at follow-up. ${ }^{\text {w8 }}$ Also, in regard to hospitalisation users must also complete a one-time registration on BMJ Learning and subsequently log in (with a BMJ Learning username and password) on every visit. 
after emergency department visits in the USA for adverse drug events in patients above 65 years, $33.3 \%$ of the 99628 hospitalisations concerned warfarin. ${ }^{\text {49 }}$ Moreover, in the general STEMI population treated with primary PCI and dual antiplatelet therapy but no anticoagulation therapy, symptomatic cerebral infarction is rare, occurring in $0.75-1.2 \%$ of all STEMI patients. ${ }^{\text {w50 }}$ Thus, the potential benefit of vitamin $\mathrm{K}$ antagonist treatment on top of dual antiplatelet therapy may not outweigh the increased bleeding risk. This calls for a randomised trial to be conducted to determine whether anticoagulation treatment prevents embolic complications in AMI patients treated with primary PCI.

Acknowledgements We gratefully acknowledge the valuable contribution of ME Hassell, MD.

Contributors RD: drafting the manuscript; FZ: critical revision; JP: interpretation of the data.

Funding This work was supported by a grant from the Dutch Heart Foundation and National Health Insurance Board/ZON MW, the Netherlands to RD. Grant number 2011 T022 + 40-00703-98-11629.

Competing interests In compliance with EBAC/EACCME guidelines, all authors participating in Education in Heart have disclosed potential conflicts of interest that might cause a bias in the article. The authors have no competing interests.

Provenance and peer review Commissioned; internally peer reviewed.

\section{REFERENCES}

1. Keren A, Goldberg S, Gottlieb S, et al. Natural history of left ventricular thrombi: their appearance and resolution in the posthospitalization period of acute myocardial infarction. J Am Coll Cardiol 1990;15:790-800

- Clinical study assessing appearance and resolution of LV thrombi on echocardiographic follow-up.

2. Asinger RW, Mikell FL, Elsperger J, et al. Incidence of leftventricular thrombosis after acute transmural myocardial infarction. Serial evaluation by two-dimensional echocardiography. N Engl J Med 1981;305:297-302.

- Eminent clinical study on the incidence of LV thrombus after AMI.

3. Visser CA, Kan G, Lie Kl, et al. Left ventricular thrombus following acute myocardial infarction: a prospective serial echocardiographic study of 96 patients. Eur Heart J 1983;4:333-7.

- Clinical study evaluating the incidence of LV thrombus formation after AMI.

4. Jugdutt BI, Sivaram CA. Prospective two-dimensional echocardiographic evaluation of left ventricular thrombus and embolism after acute myocardial infarction. J Am Coll Cardiol 1989;13:554-64.

- Clinical study evaluating LV thrombi and systemic embolism after AMI.

5. Chiarella F, Santoro E, Domenicucci S, et al. Predischarge two-dimensional echocardiographic evaluation of left ventricular thrombosis after acute myocardial infarction in the GISSI-3 study. Am J Cardiol 1998;81:822-7.

- Substudy of the GISSI-3 study providing one of the largest datasets to date on the incidence and risk factors of LV thrombus formation.

6. Solheim S, Seljeflot I, Lunde K, et al. Frequency of left ventricular thrombus in patients with anterior wall acute myocardial infarction treated with percutaneous coronary intervention and dual antiplatelet therapy. Am J Cardiol 2010;106:1197-200.

- Clinical study assessing the prevalence of LV thrombus formation in STEMI patients treated with primary PCI.

7. Mollet NR, Dymarkowski S, Volders W, et al. Visualization of ventricular thrombi with contrast-enhanced magnetic resonance imaging in patients with ischemic heart disease. Circulation 2002; 106:2873-6.

- Clinical study describing the unique position of contrast enhanced MRI in LV thrombus formation.

8. Weinsaft JW, Kim HW, Crowley AL, et al. LV thrombus detection by routine echocardiography: insights into performance characteristics using delayed enhancement CMR. J Am Coll Cardiol img 2011;4:702-12.

- Clinical study providing more insights on contrast enhanced CMR.

9. Turpie AG, Robinson JG, Doyle DJ, et al. Comparison of high-dose with low-dose subcutaneous heparin to prevent left ventricular mural thrombosis in patients with acute transmural anterior myocardial infarction. N Engl J Med 1989;320:352-7.

- Randomised controlled trial comparing high dose with low dose heparin in patients with LV thrombus formation.

10. Vecchio C, Chiarella F, Lupi G, et al. Left ventricular thrombus in anterior acute myocardial infarction after thrombolysis. A GISSI-2 connected study. Circulation 1991;84:512-19.

- Echocardiographic substudy of GISSI-2 assessing the effect of two different thrombolytic agents and heparin on the incidence and features of LV thrombi.

11. Delemarre BJ, Visser CA, Bot $\mathrm{H}$, et al. Prediction of apical thrombus formation in acute myocardial infarction based on left ventricular spatial flow pattern. J Am Coll Cardiol 1990:15:355-60.

- Clinical study assessing the predictive value of LV spatial flow pattern for thrombus formation.

12. Srichai MB, Junor C, Rodriguez LL, et al. Clinical, imaging, and pathological characteristics of left ventricular thrombus: a comparison of contrast-enhanced magnetic resonance imaging, transthoracic echocardiography, and transesophageal echocardiography with surgical or pathological validation. Am Heart J 2006;152:75-84.

- Clinical study comparing TTE, TOE and contrast enhanced MRI for the detection of LV thrombi.

13. van Dantzig JM, Delemarre BJ, Bot $H$, et al. Left ventricular thrombus in acute myocardial infarction. Eur Heart $J$ 1996;17:1640-5.

- Eminent review on diagnosis and management of LV thrombus patients.

14. Weinsaft JW, Kim RJ, Ross M, et al. Contrast-enhanced anatomic imaging as compared to contrast-enhanced tissue characterization for detection of left ventricular thrombus. J Am Coll Cardiol img 2009;2:969-79.

- Clinical study providing important information on contrast enhanced anatomic imaging compared to contrast enhanced tissue characterisation of LV thrombi.

15. Meltzer RS, Visser CA, Fuster V. Intracardiac thrombi and systemic embolization. Ann Intern Med 1986;104:689-98.

- Excellent review on intracardiac thrombi and anticoagulant treatment.

16. Johannessen KA, Nordrehaug JE, von der LG, et al. Risk factors for embolisation in patients with left ventricular thrombi and acute myocardial infarction. Br Heart J 1988;60:104-10.

- Clinical study assessing risk factors for systemic embolisation in LV thrombus patients.

17. Haugland JM, Asinger RW, Mikell FL, et al. Embolic potential of left ventricular thrombi detected by two-dimensional echocardiography. Circulation 1984;70:588-98.

- Clinical study seeking an association between echocardiographic appearances of LV thrombi and systemic embolisation.

18. Domenicucci S, Bellotti P, Chiarella F, et al. Spontaneous morphologic changes in left ventricular thrombi: a prospective two-dimensional echocardiographic study. Circulation 1987;75:737-43.

- Clinical study with serial echocardiographic studies demonstrating spontaneous morphologic changes in LV thrombi.

19. Vaitkus PT, Barnathan ES. Embolic potential, prevention and management of mural thrombus complicating anterior myocardial infarction: a meta-analysis. J Am Coll Cardiol 1993;22:1004-9.

- Relevant meta-analysis regarding the embolic potential and management of LV thrombus.

20. Stratton JR, Ritchie JL. 111 In platelet imaging of left ventricular thrombi. Predictive value for systemic emboli. Circulation 1990;81:1182-9

- Clinical study with indium-111 platelet imaging of LV thrombi. 